REOP. Vol. 21, n 1, 1er cuatrimestre, 2010. pp. 71-80

\title{
¿NECESITAN LAS UNIVERSIDADES ESPAÑOLAS UNA PRUEBA DE ACCESO INFORMATIZADA? EL CASO DE LA DEFINICIÓN DEL CONSTRUCTO Y LA PREVISIÓN DEL EFECTO EN LA ENSEÑANZA (WASHBACK) PARA IDIOMAS EXTRANJEROS
}

\author{
DO SPANISH UNIVERSITIES NEED A COMPUTER BASED ENTRANCE \\ EXAMINATION? THE CASE OF THE CONSTRUCT DEFINITION, AND THE PREVIEW \\ OF IT EFFECT IN THE TEACHING (WASHBACK) OF FOREIGN LANGUAGES
}

Jesús García Laborda Universidad de Alcalá de Henares

\section{RESUMEN}

Recientes trabajos en informática educativa en España han planteado la posibilidad real de automatizar e incluir tareas orales en la Prueba de acceso a la universidad ( $P$.

A. U.) especialmente en lenguas extranjeras. De hecho, su efecto en la docencia ya ha mostrado indicadores de sus beneficios (Glover, 2006). Desde la perspectiva de la orientación escolar esto supone una evolución significativa para muchos estudiantes tanto si van a seguir unos estudios reglados superiores como si se incorporan al mundo laboral al completar su formación escolar. La nueva prueba permitiría educar tanto estudiantes como trabajadores cualificados en lenguas extranjeras cosa que hasta ahora se ha mostrado complicada a pesar de los esfuerzos ministeriales y la insistencia de la mayor parte de los psicopedagogos residentes en los centros escolares. Sin embargo ¿Cuál sería constructo del nuevo examen? ¿Por qué supone un cambio tan profundo? Este artículo promueve la discusión sobre el constructo, la investigación nacional, los estudios y las opiniones científicas sobre este cambio. El artículo concluye que aunque una P.A.U. automatizada es un desafío significativo, aún es necesario redefinir su constructo y propone una agenda de estudio abierta a los investigadores y profesores españoles.

Palabras clave: Examen de entrada a la Universidad, exámenes de idiomas, constructor, lengua extranjera.

\begin{abstract}
Recent papers in educational computers in Spain have suggested the possibility of automatizing and including oral tasks in the University Entrance Examination (P.A.U.) specifically in the foreign language paper. In fact, its effects on teaching, has already shown indicators of its benefits (Glover, 2006). From the perspective of school orientation, this is a significant evolution for many students either if they continue in higher education or if they get in the working world after their school education.

* Doctor en Filología inglesa y Doctor en CC de la Educación. En la actualidad sus intereses son la evaluación de lenguas extranjeras, la enseñanza de idiomas por ordenador y la educación bilingüe. Ha escrito para revistas como Computers \& Education, Eurasian Journal of Educational Sciences, Language Learning \& Technology, y muchas otras. En la actualidad trabaja en un proyecto para automatizar la Prueba de Acceso a la Universidad (proyecto PAUER HUM2007-66479-C02-01/FILO). E-mail.jesus.garcialaborda@uah.es
\end{abstract}


The new test would permit to educate both students and workers qualified in foreign languages. This has been uneasy until now despite the Ministry's efforts and the insistence of most in-school educational psychologists. However, what would be the new construct? What are the implications of such a deep change. This paper provides a discussion ground on the construct and national research, studies, and scientific opinions on this change. The paper concludes that although the computer based P.A.U. test is a sound challenge, it is necessary to redefine the test construct and proposes a study agenda open to the Spanish researchers and teachers.

Key words: University entrance examination, language testing, construct, educational orientation- foreign language.

\section{Introducción}

La P.A.U. se caracteriza por una sorprendente falta de estudios considerando su importancia vital para muchos estudiantes ${ }^{1}$ aunque la sociedad y la psicología educativa demanden controles del proceso evaluativo del sistema educativo. Una simple búsqueda del término "P.A.U." en bases de datos como CINDOC o DIALNET muestra a las claras que existe una clara disociación entre los estudios en evaluación realizados y el examen de mayor impacto educativo en España. Hoy por hoy, sabemos que el efecto de la evaluación educativa determina en buena parte las recomendaciones de los orientadores de los centros y, en última instancia, incluso las decisiones de los estudiantes sobre su continuidad en la educación o su incorporación al mundo laboral (Kruger, L. J. and Shriberg, 2007) aunque también es la función escolar psicopedagógica moderar y controlar que ciertos procesos nocivos (como la orientación exclusiva a un examen) dominen al proceso educativo (Duckworth, 2009) así como observar si los procesos implicados en el uso de ordenadores para la evaluación favorecen a los alumnos (Akdemir y Oguz, 2008).

En lo referente a la prueba de inglés, García Laborda (García Laborda, 2006a) resumió la mayor parte de los realizados en los últimos 10 años y junto a Whittaker recuerdan que es necesario evaluar la necesidad de modificar y actualizar la prueba actual (Whittaker, 2006). La carencia investigadora se debe a cuatro factores: 1) falta de recursos económicos para realizar estudios fiables; 2) delegación de responsabilidad en las universidades que no publican dichos trabajos; 3) dificultades en la obtención de datos debido a la escasez de estudiosos en el tema y poca transparencia en los procesos de corrección; 4) ausencia de coordinación entre los gabinetes de orientación educativa y los responsables de la organización de la P.A.U. y 5) procesos no rigurosos en el control de calidad de los distintos estamentos que participan en el proceso de realización del examen.

Todos estos aspectos son fundamentales si se desea saber realmente el nivel de conocimientos de los estudiantes a su llegada al examen. Su capitalidad es también significativa para promover cambios y mejoras en el examen. Así, en estas circunstancias, resulta difícil fundamentar el proceso de transformación y modernización del examen, especialmente de la prueba de idiomas en la cual nos centraremos desde este momento. Por tanto, la

\footnotetext{
${ }^{1}$ Aunque en los últimos años la capacidad de admisión de la mayoría de las carreras ha restado una gran parte de la importancia que el examen de P.A.U. tuvo antaño.
} 
evolución del examen significaría la promoción de los futuros estudiantes o trabajadores permitiendo aumentar significativamente sus competencias discente-profesionales.

\section{Justificando una P.A.U. informatizada}

En 2004, García Laborda proponía la posibilidad de crear una herramienta informática que permitiese realizar exámenes orales y escritos asistidos por ordenador. Subsiguientes investigaciones y aplicaciones del mismo afirmaban haber desarrollado un prototipo que está en periodo de experimentación (García Laborda, 2006b). Dicho prototipo ha seguido un periodo de validación complejo pero positivo (García Laborda y Magal Royo, 2007). En la actualidad, esta herramienta ya realiza exámenes orales y escritos asistidos por ordenador y parece una herramienta alternativa para sustituir 0 mejorar el actual sistema utilizado para la P.A.U.

La P.A.U., tal y como es en la actualidad, es un modelo antiguo, especialmente en ciertas asignaturas como las lenguas extranjeras (Martín Úriz y Whittaker, 2005), a pesar de contar con el apoyo de cualificados investigadores (Herrera Soler, 2005). Esto, en casos precedentes, ha llevado a un efecto docente negativo tal y como lo han mostrado estudios como los de Wall y Alderson (1993) o Wall (2005 en Sri-Lanka.

Por otro lado, de acuerdo a varios autores, los exámenes asistidos por ordenador suponen las siguientes ventajas (García Laborda, 2007):

a. Reducción de las distancias: En la actualidad son muchos los alumnos podrían hacer la P.A.U. de manera sencilla sin salir de su propio centro de estudios.

b. Flexibilidad: Los exámenes asistidos por ordenador permiten gran variedad de ejercicios actualmente no reflejados en las pruebas de acceso a la universidad.

c. Inclusión de repertorios audiovisuales (multimedia): Estos repertorios añaden realismo y promueven mejores respuestas de los alumnos a través del input de los diálogos grabados y la contextualización de las preguntas por medio de imágenes y, especialmente, vídeos.

d. Integración de tareas: Estos exámenes permiten integrar varias tareas (audición, lectura, expresión, etc.) para la realización de una tarea global.

Por todas estas circunstancias y por el coste reducido de personal necesario para realizar evaluaciones más completas, existe una tendencia generalizada a realizar los exámenes con gran número de alumnos e implicación social a través de plataformas asistidas por ordenador (Alderson, 2000).

\section{¿Cómo debería ser la P.A.U. asistida por ordenador?}

El constructo de un examen es fundamental para su validez y utilidad. Puesto que la P.A.U. es un examen institucionalizado y criterial, mide las capacidades en lengua extranjera que se precisan de un alumno universitario 
para poder realizar su actividad social y académica (criterio) y que tienen una serie de características que lo definen:

a- Se barema a los estudiantes en relación no a sus conocimientos absolutos sino en relación a un nivel que se considera adecuado al final de sus estudios. Para lenguas extranjeras, un nivel intermedio (en la actualidad, solamente leído y escrito) o B1(en el Marco de Referencia Común Europeo).

b- Los exámenes deben moderarse para ser relativamente simples en sus contestaciones porque debe haber unos resultados homogéneos, justos y los resultados deben entregarse muy pocos días después.

c- Prevalecen tareas muy mecánicas basadas en respuestas cortas, de selección múltiple y la prueba más comunicativa resulta ser una redacción sobre un tema familiar al estudiante de una cantidad muy limitada de palabras.

d- El examen de cada materia se ve dentro de un conjunto mayor de exámenes con las mismas limitaciones que la suma de todas las pruebas de cada asignatura.

En resumen, el examen actual se ve restringido por un constructo no muy claramente delimitado pero usado tradicionalmente en exámenes de nivel nacionales similares en otros países europeos.

\section{La interpretación de la P.A.U. automatizada}

La interpretación de los resultados depende fundamentalmente de dos factores: la actuación de los estudiantes y el tipo de tareas que se les han requerido. Ambos se sitúan en lados opuestos de un proceso que incluye aspectos de diseño y control interno y externo de la prueba (véase Figura 1).

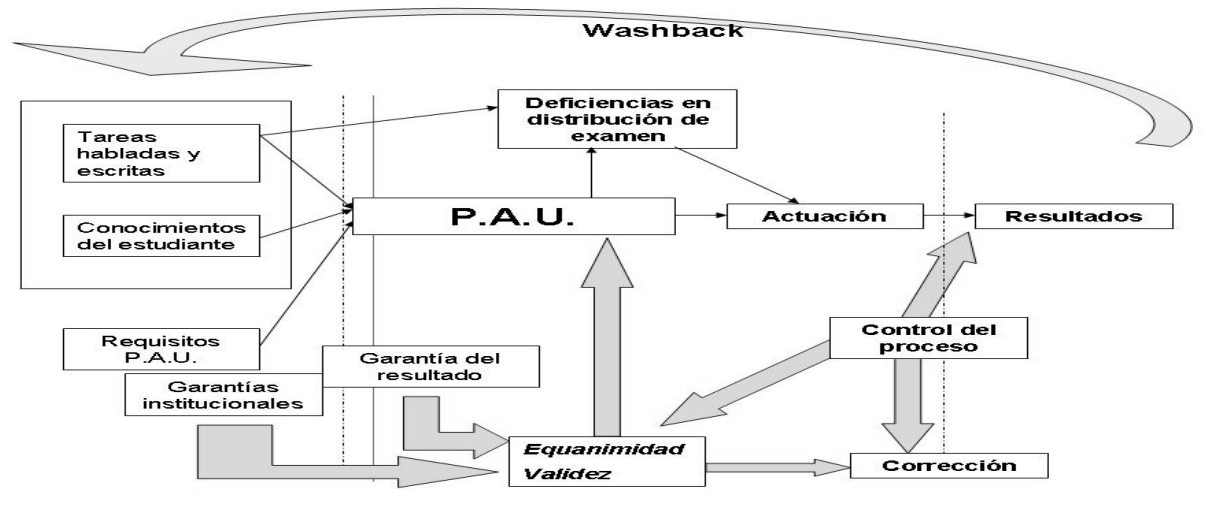

FIGURA 1.

Factores que inciden en el constructo de la P.A.U. automatizada

Según este esquema los factores iniciales del tipo de tareas y los conocimientos de los estudiantes que finalmente afectarán la actuación del estudiante y sus resultados se sitúan en los extremos opuestos mientras que, en la actualidad, la centralidad del diagrama se establece en el examen mismo 
de la P.A.U. Sin duda, se debe a que es un examen de criterio, es decir, en el que los alumnos demuestran simplemente su nivel en relación a un umbral de conocimientos (frente a los normativos que establecen comparaciones entre los alumnos que lo hacen y que claramente incidiría en la equidad y justicia de la evaluación). En casos como la P.A.U., son necesarios unos criterios de ecuanimidad y validez que incidan en la corrección para garantizar los resultados tanto en la validez de que los contenidos que se evalúan (content validity) como de sus tareas (face validity). De acuerdo a García Laborda (2006a) el tipo de tareas e ítems incluidos en el examen por las distintas universidades varían de manera significativa. Desde el diseño de las tareas (por ejemplo, cómo se presenta una tarea de transformación gramatical) a la inclusión de tipos de ítems no compartidos por varias comunidades (como la comprensión auditiva en Cataluña y, desde 2007, Galicia).

¿Cómo afectan las tareas actuales la inferencia conocimientos de los alumnos?

Un examen debe justificar su misma existencia basándose en que sus resultados sean claros indicadores de los conocimientos del alumno (test validity) (Kenyon y Van Duzer, 2003). Una P.A.U. que no contempla destrezas tanto orales como escritas presentará limitaciones en el diagnóstico y generalización de los resultados y en la validez de los mismos dentro del siguiente esquema (véase Figura 2):

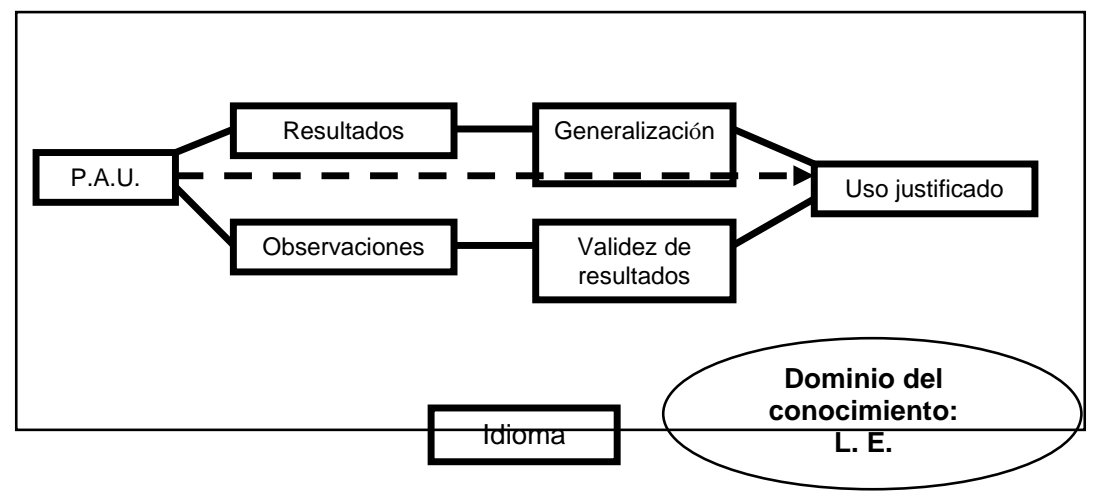

FIGURA 2.

Validez y justificación del uso de un examen

Asimismo, al contrario de lo que parece suceder en la actualidad, es necesaria una recogida de observaciones por parte de todos los sujetos que participan en el proceso: alumnos, correctores, coordinadores, gestores e, incluso, los profesores que imparten la asignatura. Estas observaciones se debe referir a cualquier aspecto de la realización del examen: factores ergonómicos, dificultad de las tareas, distribución y temporalización de las mismas, análisis de dificultades generales (y dificultad de los ítems), ajuste de la mayor parte de los alumnos a la norma deseada (o lo que es lo mismo, una distribución equilibrada del éxito y fracaso en el examen). Esas observaciones deben llevar a extrapolar y contrastar esos resultados con el nivel de conocimiento demandados por los administradores. Por ejemplo, contrastar los resultados de P.A.U. con los de otros exámenes como TOEFL o aquellos de Cambridge Board of Exams. 


\section{Definiendo un constructo para una P.A.U. automatizada}

Una de las funciones del constructo es sostener y dar validez a un examen (Fulcher y Davidson, 2007). Por eso, el constructo de la P.A.U. asistida por ordenador debe estar abierto a la mayor cantidad y variedad de tipos de pruebas e ítems (Brennan, 2001). En la actualidad este constructo se ve limitado por el tiempo en la realización y corrección, y el hecho de que no es la única asignatura del examen.

En los últimos años se han prodigado los exámenes normativos de gran impacto automatizados (Roever, 2006) cuyos constructos varían según sus objetivos y las necesidades de la evaluación. García Laborda (2006c) señalaba que una plataforma informática permite una gran flexibilidad de tareas (Tabla $3)$.

TABLA 3: Tipos de tareas y su corrección en una P.A.U. automatizada

\begin{tabular}{|c|c|c|}
\hline Destreza & Tipos de ejercicio & Tipo de corrección \\
\hline Comprensión lectora & $\begin{array}{l}\text { Título justificado } \\
\text { Preguntas abiertas } \\
\text { Definiciones de palabras } \\
\text { Verdadero / Falso } \\
\text { Resumen de texto } \\
\text { Relacionar } \\
\text { Sinónimos } \\
\text { Elección múltiple } \\
\text { Rellenar huecos }\end{array}$ & $\begin{array}{l}\text { Corrección } \\
\text { automática } \\
\text { Intervención humana } \\
\text { Intervención humana } \\
\text { Corrección } \\
\text { automática } \\
\text { Intervención humana } \\
\text { Corrección } \\
\text { automática } \\
\text { Corrección } \\
\text { automática } \\
\text { Corrección } \\
\text { automática } \\
\text { Corrección } \\
\text { automática }\end{array}$ \\
\hline $\begin{array}{l}\text { Conocimientos } \\
\text { gramaticales }\end{array}$ & $\begin{array}{ll}\begin{array}{l}\text { Modificaciones } \\
\text { (varios) }\end{array} & \text { gramaticales } \\
& \end{array}$ & $\begin{array}{l}\text { Corrección } \\
\text { automática }\end{array}$ \\
\hline Producción oral & Identificación fonética & Modelo predefinido \\
\hline Producción escrita & Redacción & Intervención humana \\
\hline \multirow[t]{2}{*}{ Comprensión oral } & Relacionar & $\begin{array}{l}\text { Corrección } \\
\text { automática }\end{array}$ \\
\hline & Elección múltiple & $\begin{array}{l}\text { Corrección } \\
\text { automática }\end{array}$ \\
\hline
\end{tabular}

Llegados a este punto es necesario sugerir un constructo para la P.A.U. automatizada.

Este constructo quedará definido en relación a una serie de parámetros:

Evaluación del grado de confianza del examen: Para ello, el propósito y los objetivos principales y secundarios de las pruebas de producción y comprensión oral y escrita deben quedar claramente definidos y expresados. A través de una P.A.U. automatizada deberíamos llegar a que el alumno pueda interpretar el ínput externo a través de repertorios audiovisuales y pueda expresar de manera oral y escrita mensajes apropiados a un estudiante universitario.

Evaluación del grado de interactividad: Con esta evaluación se mide si el alumno es capaz de realizar el examen sin mayores dificultades que aquellas limitaciones propias de los conocimientos (o su ausencia) de lengua extranjera. Por ejemplo, algunos exámenes actuales presentan ciertas dificultades ya que 
tienen interfaces inusuales para estudiantes españoles o requieren teclados especiales, etc. Como consecuencia, el alumno no solamente tiene que demostrar su competencia lingüística sino su pericia informática.

Evaluación del grado de autenticidad: En este caso se refiere al hecho de que el examen debe reflejar los conocimientos reales del alumno. Amengual Pizarro (2006) propone que esto se puede realizar a través de una validación interna pero, sin duda, debe haber una externa como un contraste con TOEFL o IELTS.

Evaluación del grado de impacto: Aunque el impacto ha sido visto al principio de este artículo recordaremos que el impacto debe ser justo y equiparar a los alumnos de las distintas universidades del país ya que los alumnos pueden seguir estudios en cualquiera de las mismas. Desafortunadamente, García Laborda (2006a) ha presentado indicios de que esto no es así.

Evaluación de practicidad: Este aspecto se refiere a si las interpretaciones de los resultados dan verdadera idea de las fuerzas y debilidades del alumno. En la actualidad, la P.A.U. es tan limitada en cuanto a extensión, medidas, y tipos de pruebas que parece difícil creer que sea práctica. El aumento de tipos de tareas y la creación de nuevos tipos de ítems permitiría aumentar el grado de practicidad.

Sin duda, una plataforma informática y la automatización del examen producirían grandes cambios en la forma en que se imparten las clases, el contenido de los libros de texto y las estrategias de examen utilizadas para la realización del mismo. Estos cambios también deben reflejar el constructo y los criterios de evolución del mismo.

\section{Efectos de la P.A.U. asistida por ordenador}

Una P.A.U. asistida por ordenador tal y como se ha descrito más arriba tendría un efecto concatenado que va desde la realización del propio examen a la obtención de datos y descubrimientos de investigación. La realización del examen conllevaría la obtención de datos cuantitativos (las notas) y cualitativos (las reflexiones de los alumnos al final del ejercicio) que mueven tanto a los profesores como a los administradores a realizar cambios y mejoras en todo el proceso. Como resultado aparecerían modificaciones en el examen (generalmente concertados en una reunión anual que se suele celebrar entre los profesores responsables de P.A.U. y los profesores de enseñanza secundaria). También habría, sin duda, variaciones en la metodología usada en impartir la lengua extranjera en Bachillerato adaptando los tipos de tareas, extensión de los ejercicios, registros de lectura y otros aspectos que, generalmente, son menores.

Tal y como ha sugerido García Laborda (2006b) el proceso de incorporar una prueba oral incidiría en la necesidad de realizar una mayor cantidad de práctica hablada y, sin duda, eso incidiría en el desarrollo de habilidades orales que en la actualidad se encuentran bastante limitados.

Los niveles y estrategias cognitivas influyen en la forma en que se realiza el examen. Por ejemplo, si creemos que los estudiantes deben ser 
capaces de tomar notas mientras asisten a una conferencia o si los alumnos deben poder interpretar diagramas relacionados con su especialidad a simple vista, el examen deberá incluir tareas que se fundamenten en dichas posibilidades. De esto se deduce que los niveles cognitivos también afectan al constructo aunque son difícilmente mensurables de manera psicométrica. El constructo, por tanto, medirá los conocimientos lingüísticos, las estrategias y procedimientos deseados en un estudiante universitario y las características de las tareas que se piensa que los alumnos deben poder realizar. La propuesta actual en una plataforma automatizada de examen sería:

FIGURA 4: Aspectos del constructo y su reflejo en las tareas de una P.A.U. asistida por ordenador

\begin{tabular}{|c|c|c|}
\hline $\begin{array}{l}\text { Aspectos del } \\
\text { constructo }\end{array}$ & Capacidades deseables & Reflejo en tareas de P.A.U. \\
\hline $\begin{array}{l}\text { Tareas } \\
\text { lingüísticas }\end{array}$ & $\begin{array}{l}\text { La mayoría miden el uso } \\
\text { de la segunda lengua, } \\
\text { registros de estilo y } \\
\text { capacidad de lectura }\end{array}$ & $\begin{array}{l}\text { - Selección múltiple y completar huecos } \\
\text { - Responder a preguntas de lectura por cualquiera de } \\
\text { los medios actuales más preguntas abiertas. } \\
\text { - Comprensión de textos orales y respuesta a los } \\
\text { mismos. } \\
\text { - Poder contestar preguntas orales de manera } \\
\text { sencilla y efectiva. Poder hacer descripciones } \\
\text { orales básicas (ninguna de estas tareas se hace en } \\
\text { la actualidad) }\end{array}$ \\
\hline $\begin{array}{l}\text { las estrategias } \\
\text { y } \\
\text { procedimientos }\end{array}$ & $\begin{array}{l}\text { Ser capaz de realizar } \\
\text { actividades intelectuales } \\
\text { deseadas en un } \\
\text { estudiante universitario }\end{array}$ & $\begin{array}{l}\text { Tareas con toma de datos como pequeñas } \\
\text { explicaciones en segunda lengua, lectura de amplios } \\
\text { textos contextualizados, comprensión de diagramas en } \\
\text { un tiempo corto de tiempo. }\end{array}$ \\
\hline $\begin{array}{l}\text { Madurez } \\
\text { discente }\end{array}$ & $\begin{array}{l}\text { Toma de posiciones crítica } \\
\text { ante los contenidos que se } \\
\text { presentan }\end{array}$ & $\begin{array}{l}\text { Tareas de opinión y comprensión. Podría incluir } \\
\text { ensayos sobre posicionamiento del alumno ante } \\
\text { recursos audiovisuales o pequeñas explicaciones. }\end{array}$ \\
\hline
\end{tabular}

\section{Conclusiones y líneas de investigación}

En general cabe esperar que el proyecto Paulex ${ }^{2}$ (subvencionado por el MEC) estudien y demuestren cuestiones en torno a 4 puntos principales:

a) Que un constructo mejor definido y que incluya todas las destrezas de la lengua beneficia por igual a todos los alumnos, motiva su mejorar personal y es equitativo siempre que los docentes se centren en los contenidos por encima de las estrategias del examen. mismo.

b) Que la inversión necesaria para el cambio tecnológico justificaría el

c) Que los estudiantes y profesores pueden aceptar cambios tan significativos en el constructo y tareas del examen mismo. Secundariamente habría que medir sus reacciones, pasividad o, incluso, rechazo ante el mismo.

d) Que una mayor implicación de todos los aspectos cognitivos y madurativos de los alumnos se refleja en unos resultados acordes en la

\footnotetext{
${ }^{2}$ El proyecto Paulex Universitas está subvencionado por el plan nacional de investigación del Ministerio de Educación de España (HUM2007-66479-C02-01/FILO) de 2007 y dirigido por la Dra. Emilia Enríquez Carrasco.
} 
población estudiantil que se enfrenta a la P.A.U. cada año. La coherencia entre los resultados de las distintas asignaturas debería ser también una prioridad.

A modo de conclusión diremos que queda abierto el debate en cuanto al constructo de la P.A.U. asistida por ordenador, en nuestro caso, especialmente en lo referente a la asignatura de lengua extranjera (especialmente, del inglés). En caso de que sea viable es de suponer que la interpretación de los resultados muestre una estrecha relación con el constructo del examen y que ayude a identificar al alumno que supere la P.A.U. como maduro y capaz para enfrentarse a sus estudios universitarios con garantía de éxito. Esta suposición debe tener, ciertamente, un apoyo en la investigación psicométrica y tratar de hacer un seguimiento de la actuación de los alumnos durante sus estudios universitarios, algo que, hasta la fecha, parece necesitar cierto interés ministerial del que carece. Por tanto, queda en manos, de la cooperación entre los educadores, psicopedagogos y las autoridades educativas la creación de buenas prácticas que conduzcan a una puesta en funcionamiento de tan revolucionario sistema con un papel muy especial para la psicopedagogía que se tendrá que hacer cargo, quizás algún día, de velar por la moderación y disminuir los efectos negativos de los exámenes de alto impacto en los estudiantes (Putwain, 2008; Sackett, Borneman y Connelly, 2008).

\section{Referencias bibliográficas}

Akdemir, O., y Oguz, A. (2008). Computer-based testing: An alternative for the assessment of turkish undergraduate students. Computers \& Education, 51(3), 1198-1204.

Alderson, J.C. (2000) Technology in testing: the present and the future, System, 28(4), 593-603.

Amengual Pizarro, M. (2006) Análisis de la prueba de inglés de la Universitat de les Illes Balears. Ibérica, 11, 29-60.

Brennan, R. L. (2001). Generalizability theory. New York: Springer-Verlag.

Duckworth, A. L. (2009). (Over and) beyond high-stakes testing. American Psychologist, 64(4), 279-280.

Fulcher, G. y Davidson, F. (2007). Language Testing and assessment. London: Routledge.

García Laborda, J. (2004) HIEO: Investigación y desarrollo de una Herramienta Informática de evaluación oral multilingüe, Didáctica. Lengua y literatura, 16, 77 -88 .

García Laborda, J. (2006a) Analizando críticamente la Selectividad ¿Todos los estudiantes tiene las mismas posibilidades?, Tesol Spain Newsletter, 30()3, 913.

García Laborda, J. (2006b) La Plataforma de exámenes multilingüe PREVALEX: resultados del diseño y perspectivas de investigación futura de la Plataforma de Exámenes Valenciana de Lenguas Extranjeras, Didáctica. Lengua Y Literatura, 18, 135 - 145.

García Laborda, J. (2006c) ¿Qué pueden aportar las nuevas tecnologías al examen de Selectividad de inglés? Un análisis de fortalezas y oportunidades, Revista De Ciencias De La Educación, 206, 151 - 165. 
García Laborda, J. (2007) On the net: Introducing standardized ESL/EFL exams, Language Learning And Technology, 11(2), 3-9.

García Laborda, J. y Magal Royo, T. (2007) Diseño y validación de la plataforma PLEVALEX como respuesta a los retos de diseño de exámenes de lenguas para fines específicos, Ibérica, 14, 43-59.

Glover, P. (2006). Examination Influence on How Teachers Teach: A Study of Teacher Talk. PhD tesis no publicada, Department of Linguistics and Modern English Language, Lancaster University, Lancaster, England.

Herrera Soler, H. (2005) El test de elección múltiple: herramienta básica en la Selectividad, en Herrera Soler, H y García Laborda, J. (EDS.) Estudios y Criterios Para Una Selectividad De Calidad En El Examen De Inglés, Valencia: Universidad Politécnica de Valencia, 65-98.

Kenyon, D., y Van Duzer, C. (2003). Valid, Reliable, And Appropriate Assessments For Adult English Language Learners, Washington, DC, Estados Unidos: ERIC Q \& A National Center for ESL Literacy Education.

Kruger, L. J. and Shriberg, D. (2007). High stakes testing: New Challenges and Opportunities for School Psychology. Binghamton: The Haworth Press.

Martín Úriz, A. y Whittaker, R. (2005). La composición como comunicación: una experiencia en las aulas de lengua inglesa en bachillerato. Madrid: Ediciones de la Universidad Autónoma de Madrid.

Putwain, D. (2008). Do examinations stakes moderate the test anxiety-examination performance relationship? Educational Psychology, 28(2), 109-118.

Roever, C. (2006). Validation of a web-based test of ESL pragmalinguistics. Language Testing, 23(2), 229-256.

Sackett, P. R., Borneman, M. J., \& Connelly, B. S. (2008). High stakes testing in higher education and employment: Appraising the evidence for validity and fairness. American Psychologist, 63(4), 215-227.

Wall y Alderson, J. C. (1993). Examining Washback: The Sri Lankan Impact Study. Language Testing, 10()1, 41-69.

Wall, D. (2005). The impact of high-stakes examinations on classroom teaching: a case study using insights from testing and innovation theory. (Cambridge, Cambridge University Press).

Whittaker, R. (2006) Reseña de Estudios y Criterios para una Selectividad de Calidad en el Examen de Inglés. Estudios Ingleses De La Universidad Complutense, 14, 191-214.

Fecha de recepción: 18-06-2007

Fecha de revisión: 06-07-2009

Fecha de aceptación: 19-01-2010 